\title{
Radar Cross Section Reduction of Metallic Plate Loaded with Via Hole Dielectric Substrate
}

\author{
Saber H. Zainud-Deen ${ }^{1}$, Hend A. Malhat ${ }^{2}$, and Nagwa A. Shabayek ${ }^{3}$ \\ ${ }^{1}$ Faculty of Engineering and Technology, Badr University in Cairo, Egypt \\ ${ }^{2}$ Faculty of Electronic Engineering, Menoufia University, Egypt \\ ${ }^{3}$ Faculty of Electronic Engineering, Menoufia University, Egypt \\ *corresponding author, E-mail: er_honidal@yahoo.com
}

\begin{abstract}
Radar cross section (RCS) stealth technology has a major importance in the military applications. In this paper, RCS reduction of metallic sheet is investigated through loading it by dielectric substrate with tapered via holes. The via holes tapering are drilled in two approaches according to the choice of the reference hole radius. In the first approach, the via holes are tapered in a single direction from row to row and are kept fixed along the single row. A reduction on RCS of $13 \mathrm{~dB}$ below the unloaded case is achieved. The RCS reduction frequency band is shifted down by increasing the negative value of scaling parameter due to the reduction of the effective dielectric constant of the dielectric sheet. In the second approach, the holes are tapered along the off-diagonal directions where the tapering occurs along both row and column. A symmetrical structure around the main diagonal is produced. The reduction in RCS is achieved with the band from 11 to $12.75 \mathrm{GHz}$ for different scaling coefficient values with best RCS reduction of $33.5 \mathrm{~dB}$ occurs at $\alpha=0.18$. Symmetrical wide angle RCS reduction from $-109^{\circ}$ to $109^{\circ}$ degrees is reached.
\end{abstract}

\section{Introduction}

The radar cross section (RCS) is a measure of the detectability of an object by the radar system [1]. Theoretically, the RCS is the equivalent area of the target which produce the same quantity of reflected power that is detected back at the radar system [2]. RCS reduction plays an significant function in the stealth technology for modern warfare such as aircraft, missiles, ships, and other military vehicles [3]. Smaller RCS vehicles enhance its capability to avoid radar detection, from land-based facilities, guided weapons or other destructive vehicles. There are different factors that influence the object RCS, such as the object material, orientation, and relative size in terms of the wavelength. Also, The RCS is affected by the angles, frequency and polarization of incident and reflected waves with respect to the orientation of the object [4]. Different techniques have been investigated in literatures for RCS reduction of different objects as coated with absorbing materials (RAMs, FSS, AMC) [5], object reformation [6], and metamaterial [7-9]. RCS reduction is achieved via reducing the incident wave energy through thermal loss, spreading out, destructive interference, or reflection from the target surface. The RCS minimization based on plasma has been analyzed in [10]. Perforation technique has been used in different antenna applications, such as supporting multi-set operation, bandwidth and gain enhancement [11-13]. The perforation technique has the advantages of being all material loading with low losses, light weight, and low profile [12]. The perforation is accomplished by drilling holes in the dielectric sheet to alter its effective dielectric constant.

This paper investigates the RCS reduction from the metallic planar plate by loading it with dielectric shaped structures. A tapered drilled holes in square dielectric sheet in single and dual directions are utilized. The issue of changing the scaling factor on the RCS is studied. Different perforation configurations of the dielectric loading are demonstrated. The finite integration technique (FIT) [14] are used in simulating all proposed structures.

\section{Numerical Results}

The scattering from metallic plates with different figures and sizes have been examined and calculated in [15]. A metallic plate with an area of $380 \mathrm{~cm}^{2}$ is used to investigate the suggested RCS reduction technique. The plate is loaded with $10 \mathrm{~mm}$ thicken lossless dielectric sheet of $\varepsilon_{r}=12$. Via holes are drilled into the sheet in a predetermined pattern and is illuminated by normal $\mathrm{x}$ polarized plane wave as plotted in Fig. 1. Twenty-six by twentysix holes are drilled in the sheet with radius $R_{o}$ and separation distance, $\mathrm{S}_{\mathrm{p}}$. Each $2 \times 2$ holes have the same radii and acts as a single block where $13 \times 13$ blocks are allocated on the metallic sheet.

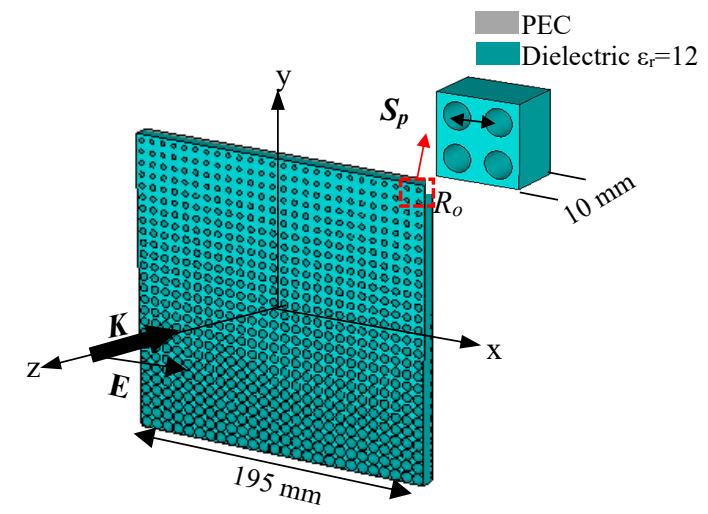

Fig. 1. The configuration of loaded metallic sheet with perforated dielectric substrate. 
The via holes changes the effective dielectric constant of the sheet, as given by [13],

$$
\begin{gathered}
\varepsilon_{\text {reff }}=\varepsilon_{r}(1-\beta)+\beta \\
\beta=\frac{\pi R_{o}^{2}}{2(\sqrt{3} / 4) S_{p}^{2}}
\end{gathered}
$$

The control of deflected beam angle from the loaded metallic plate is achieved by tapering the via holes' radii, $R_{n}$, of the reference $\mathrm{n}^{\text {th }}$ row or column or diagonal according to

$$
R_{n}=R_{o}-(n-\zeta) . \alpha \quad \text { where } n=1,2 \ldots, 13
$$

where $R_{o}$ is the radius of the reference hole, $\alpha$ is a scaling parameter and an offset coefficient, $\zeta$. The direction of holes' radii tapering is controlled by values of $\alpha, \zeta$, and the reference element in the system. The RCS reduction behavior of different hole tapering configurations is investigated. By changing the hole radii will add a progressive phase shift to the reflected plane wave along one axis of the loaded-PEC plate. This phase shift will result in deflecting beam with an angular offset from the normal charge. The offset shift in the deflected beam is frequency dependent due to the change in the effective dielectric constant, and introduces RCS decrease over a broad frequency band.
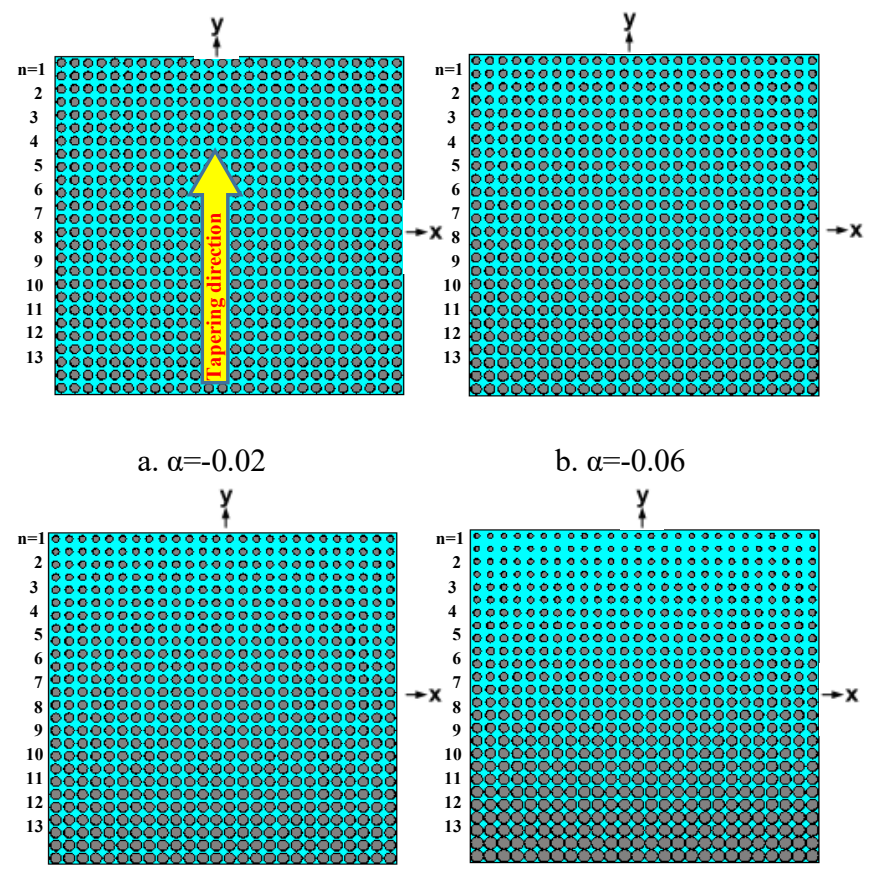

c. $\alpha=-0.1$

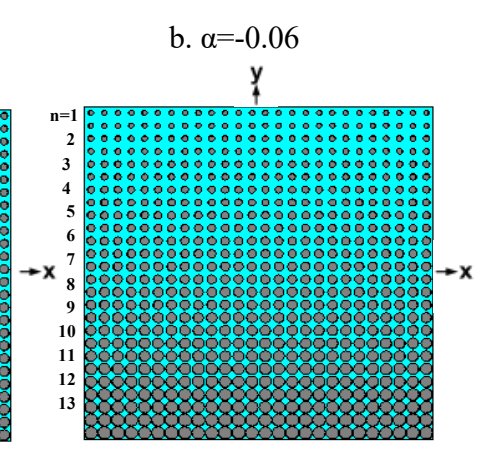

d. $\alpha=-0.18$
Fig. 2. A schematic diagram for the tapered via holes drilled in in one direction with $\alpha, R_{0}=2.64 \mathrm{~mm}, \mathrm{~S}_{\mathrm{p}}=7.5 \mathrm{~mm}, n=1,2 \ldots, 13$ and $\zeta$ $=7$ and $1^{\text {st }}$ reference element.

\subsection{Dielectric Via Hole Row Tapering in One Direction}

In this tapering configuration, two cases of holes' radii tapering according to the choice of the reference row are investigated. In the first case, the scaling parameter, $\alpha$, is selected to reduce the via hole's radii along the $\mathrm{y}$-axis while keeping it fixed along the $\mathrm{x}$-axis (i.e. from row to row). A schematic diagram for the tapered via holes drilled in the dielectric sheet for $\mathrm{S}_{\mathrm{p}}=7.5 \mathrm{~mm}, \zeta=7$, and $n=1$, $2 \ldots, 13$. The first row is chosen as a reference element with radius $R_{\mathrm{0}}=2.64 \mathrm{~mm}$ is plotted in Fig. 2. Different scaling factor values scaling coefficient $\alpha=-0.02,-0.06,-0.1$, and -0.18 are investigated.
The holes' radii are increased gradually from the top to the bottom. The electric field of the incident plane wave is normal to the direction of tapered graded holes. The frequency response of the RCS for the loaded metallic sheet with tapered graded sheet in one direction is shown in Fig. 3. The reduction in RCS is achieved using the loaded metallic sheet compared to the unloaded case. The RCS reduction frequency band is shifted down by increasing the negative value of $\alpha$ due to the reduction of the effective dielectric constant of the dielectric sheet. The frequency band from 11.5 to $13.5 \mathrm{GHz}$ has reduced RCS for different $\alpha$ values. The RCS angle variation for $\alpha=-0.18$ compared to the unloaded case at $11.75 \mathrm{GHz}$ are plotted in Fig. 4. A symmetrical pattern in the $\mathrm{x}-\mathrm{z}$ plane is obtained with wide angle RCS reduction extending from $130^{\circ}$ to $+130^{\circ}$ degrees. The unsymmetrical pattern in the y-z plane is depicted with deflected beam at $10^{\circ}$ degrees offset from the normal direction. A reduction on RCS of $13 \mathrm{~dB}$ to a lower place the unloaded case is reached. The 3D RCS patterns at $11.75 \mathrm{GHz}$ for different loading cases are illustrated in Fig. 5.

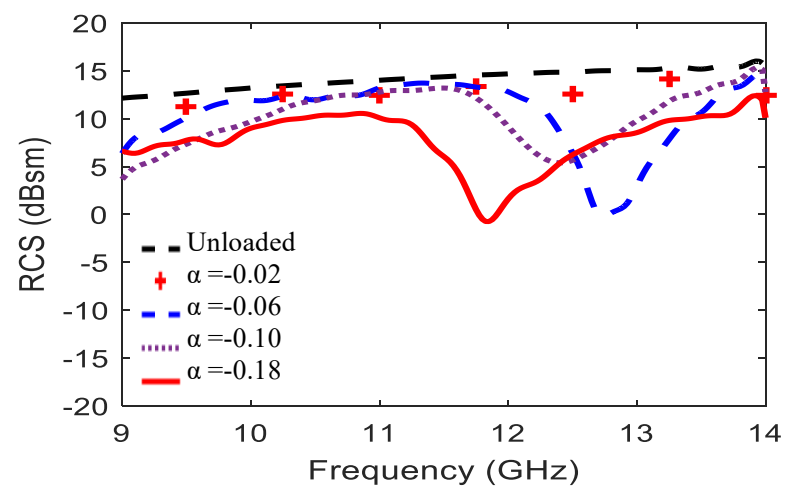

Fig. 3. The RCS versus frequency in one direction tapered holes at different scaling factor $\alpha, R_{0}=2.64 \mathrm{~mm}, \mathrm{~S}_{\mathrm{p}}=7.5 \mathrm{~mm}$, and $\zeta=7$.
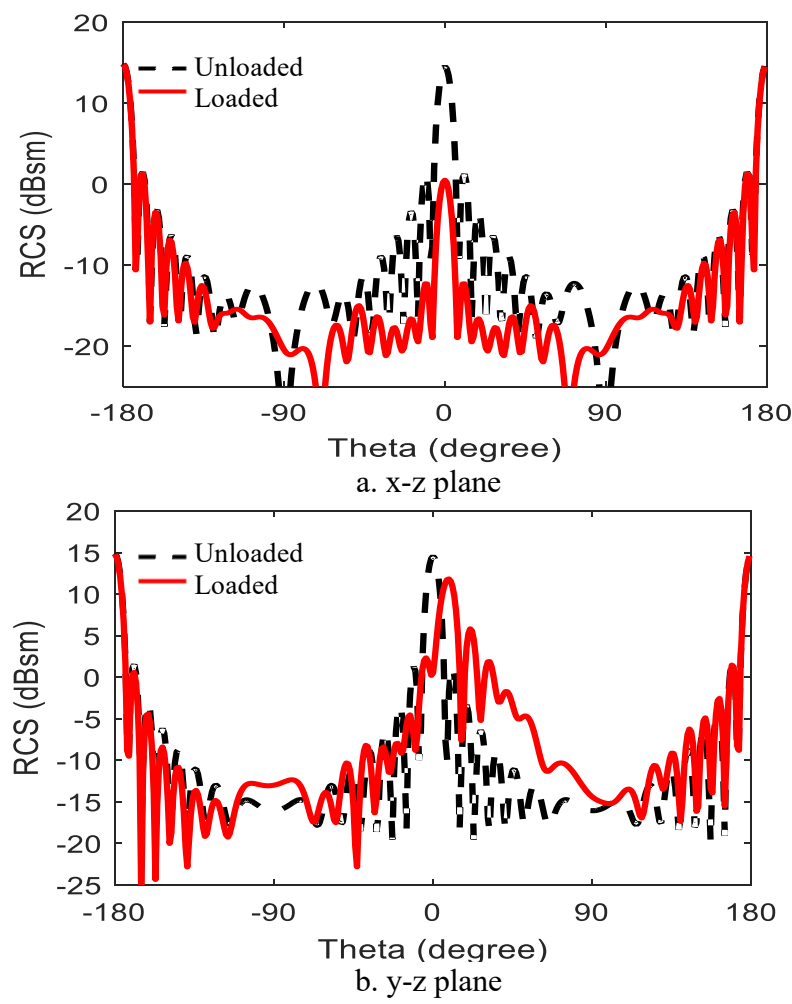

Fig. 4. The angle variation of RCS for one direction tapered holes at $\alpha=-0.18$ and $f=11.75 \mathrm{GHz}$. 


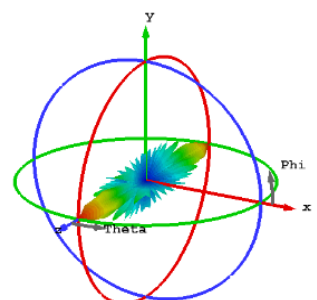

a. Unloaded case

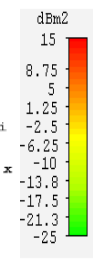

b. loaded with $\alpha=-0.18$

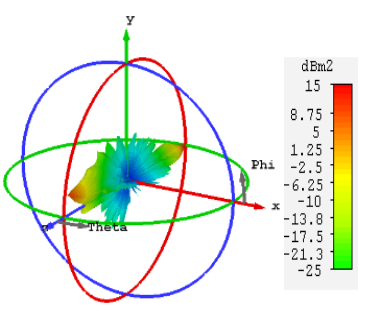

Fig. 5. The 3D RCS for unloaded and loaded metallic sheet at $11.75 \mathrm{GHz}$.

In the second case, the reference element is chosen along the central row of the drilled holes in the loaded dielectric sheet, the radii are tapered in one direction above and below this row as presented in Fig. 6. Different values of the scaling factor, $\alpha=0.02$, $0.06,-0.1$, and -0.18 are investigated with $R_{0}=2.64 \mathrm{~mm}, \mathrm{~S}_{\mathrm{p}}=7.5$ $\mathrm{mm}, n=1,2, \ldots, 7$ and $\zeta=1$. For each scaling parameter, $\alpha$, the hole's radii are increased above and below the central row while they kept fixed along each row. The RCS variation versus frequency for loading metallic sheet illuminated by an x-polarized plane wave is shown in Fig. 7a. The RCS reduction in the loaded case is accomplished along the frequency band from 9 to $14 \mathrm{GHz}$ for different $\alpha$ compared to the unloaded case. The effect of lighting up the loaded structure by a y-polarized plane wave on the RCS variations are presented in Fig. $7 b$. The value of the RCS reduction of the PEC plate loaded with tapered graded perforated dielectric sheet is increased by decreasing $\alpha$ in the band from 9 to $14 \mathrm{GHz}$.

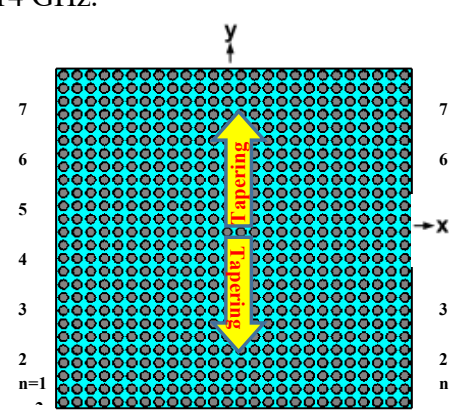

a. $\alpha=-0.02$

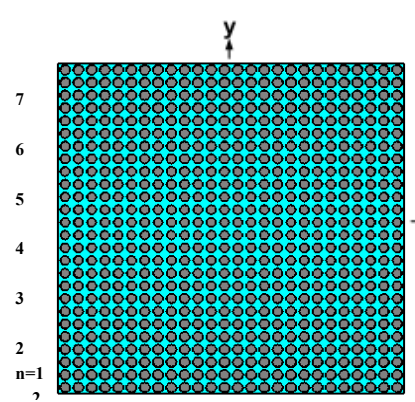

c. $\alpha=-0.1$

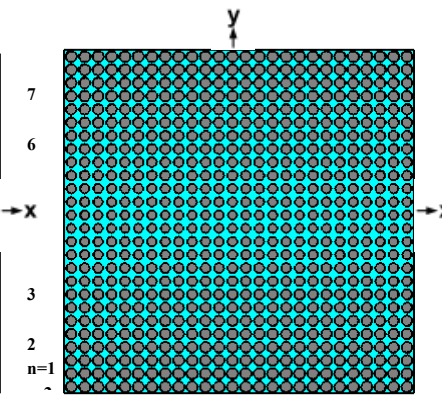

b. $\alpha=-0.06$

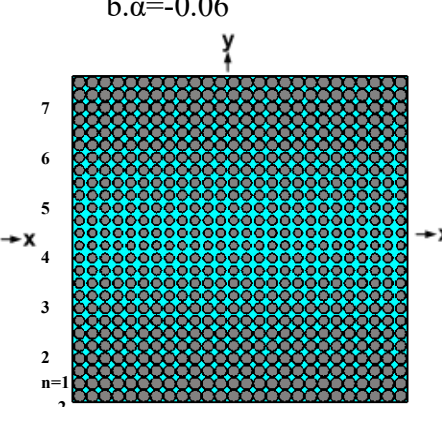

d. $\alpha=-0.18$
Fig. 6. The tapered holes from the central row-in one directions with different scaling coefficient, $\alpha, \mathrm{R}_{0}=2.64 \mathrm{~mm}, \mathrm{n}=1,2 \ldots, 7$ and $\zeta=1$.

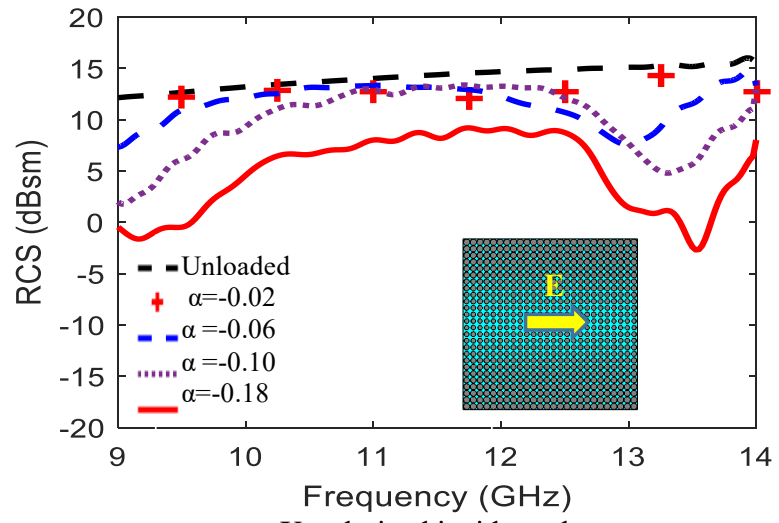

a. X-polarized incident plane wave.

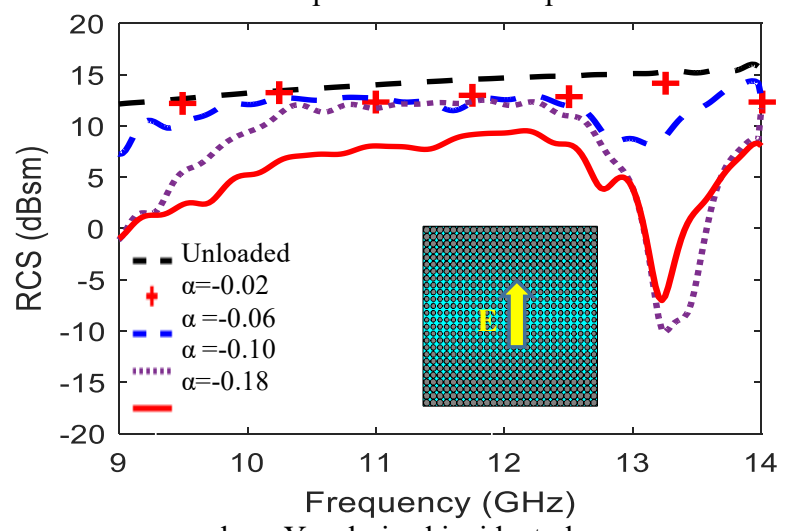

b. Y-polarized incident plane wave

Fig. 7. The RCS response for tapered holes from the central row-in one directions with different scaling coefficient, $\alpha R_{0}=2.64 \mathrm{~mm}$, and $\zeta=1$ for.

\subsection{Dielectric Via Hole Diagonal Tapering in Two Direction}

Two directions via hole tapering is achieved by drilling the loading dielectric substrate along the off-diagonals directions. Two cases of off-diagonal tapering are studied. The first case starts with large hole radius of $R_{o}=2.64 \mathrm{~mm}$ along the substrate main diagonal, and gradually decrease the other off-diagonals holes' radii according to the scaling parameters $\alpha$ where $S_{p}=7.5 \mathrm{~mm}, n=1$, $2 \ldots, 13$ and $\zeta=1$ as shown in Fig. 8. The hole's radii are decreased symmetrically along each row and column above and under the main stroke. A symmetrical structure around the main diagonal is produced. The scattered RCS variations versus frequency for loading metallic sheet illuminated by x-polarized and y-polarized incident plane wave is shown in Fig. 9a and Fig. 9b respectively. The RCS reduction is enhanced by increasing the scaling parameter, $\alpha$ compared to the unloaded case. The reduction in RCS is achieved with the band from 11 to $12.75 \mathrm{GHz}$ for different scaling coefficient values with best RCS reduction occurs at $\alpha=0.18$. Symmetrical results are obtained for both $\mathrm{x}$ - and $\mathrm{y}-$ polarized plane wave due to the structure symmetry. The angle variation of RCS for $\alpha=0.18$ at $11.75 \mathrm{GHz}$ are shown in Fig. 10 . Symmetrical patterns in different planes are obtained with wide angle RCS reduction from $-109^{\circ}$ to $109^{\circ}$ degrees by $33.5 \mathrm{~dB}$ is achieved. 3D RCS scattering patterns at different scaling factor $\alpha$ and $f=11.75 \mathrm{GHz}$ are illustrated in Fig. 11 . 


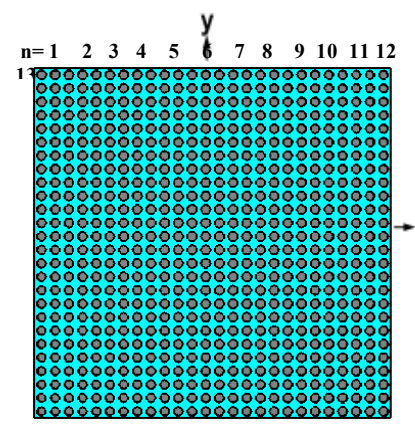

a. $\alpha=0.02$

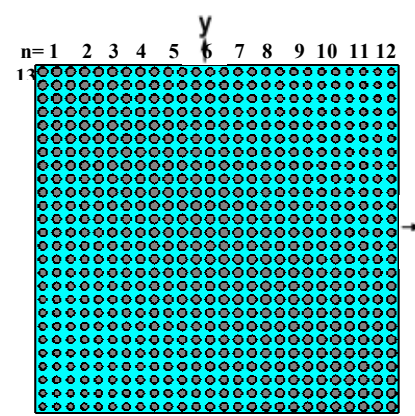

c. $\alpha=0.1$

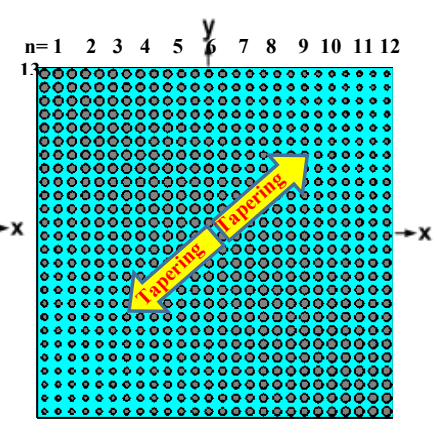

b. $\alpha=0.06$

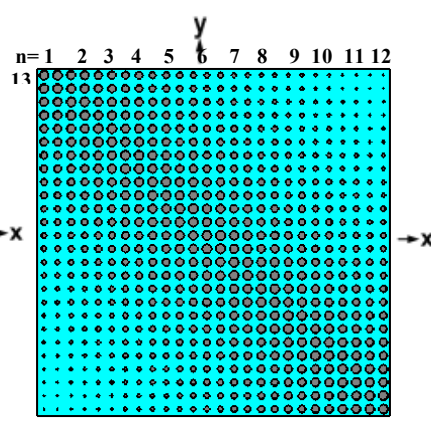

d. $\alpha=0.18$
Fig. 8. The two dimensions, holes tapering with different $\alpha, R_{0}=$ $2.64 \mathrm{~mm}, \mathrm{~S}_{\mathrm{p}}=7.5 \mathrm{~mm}, n=1,2 \ldots, 13$ and $\zeta=1$.

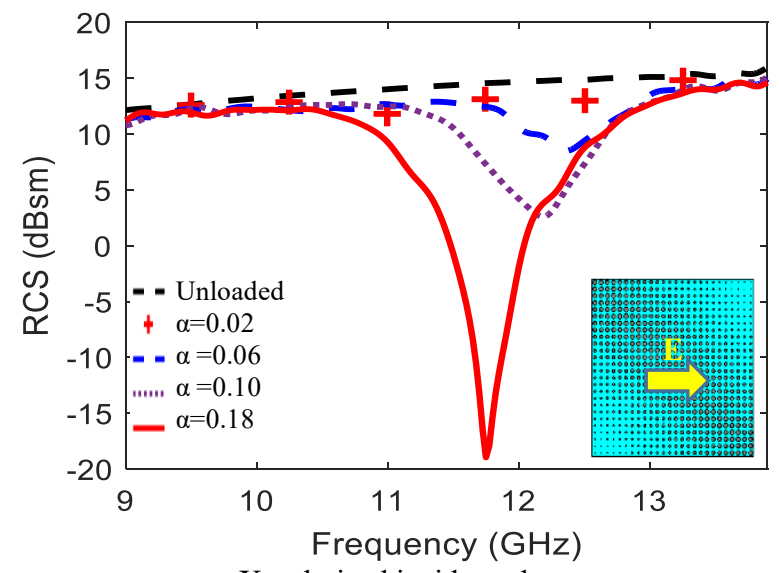

a. X-polarized incident plane wave.

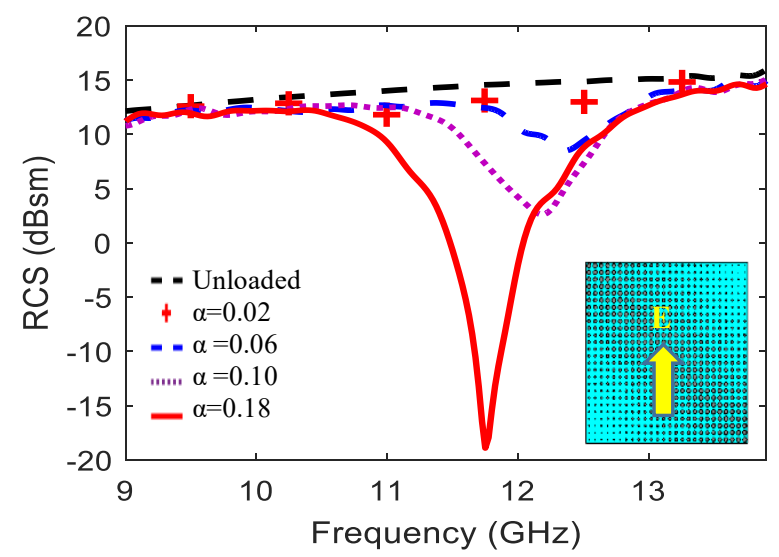

b. Y-polarized incident plane wave.

Fig. 9. The RCS versus frequency for two dimension holes tapering off-diagonal with different $\alpha, R_{o}=2.64 \mathrm{~mm}$.
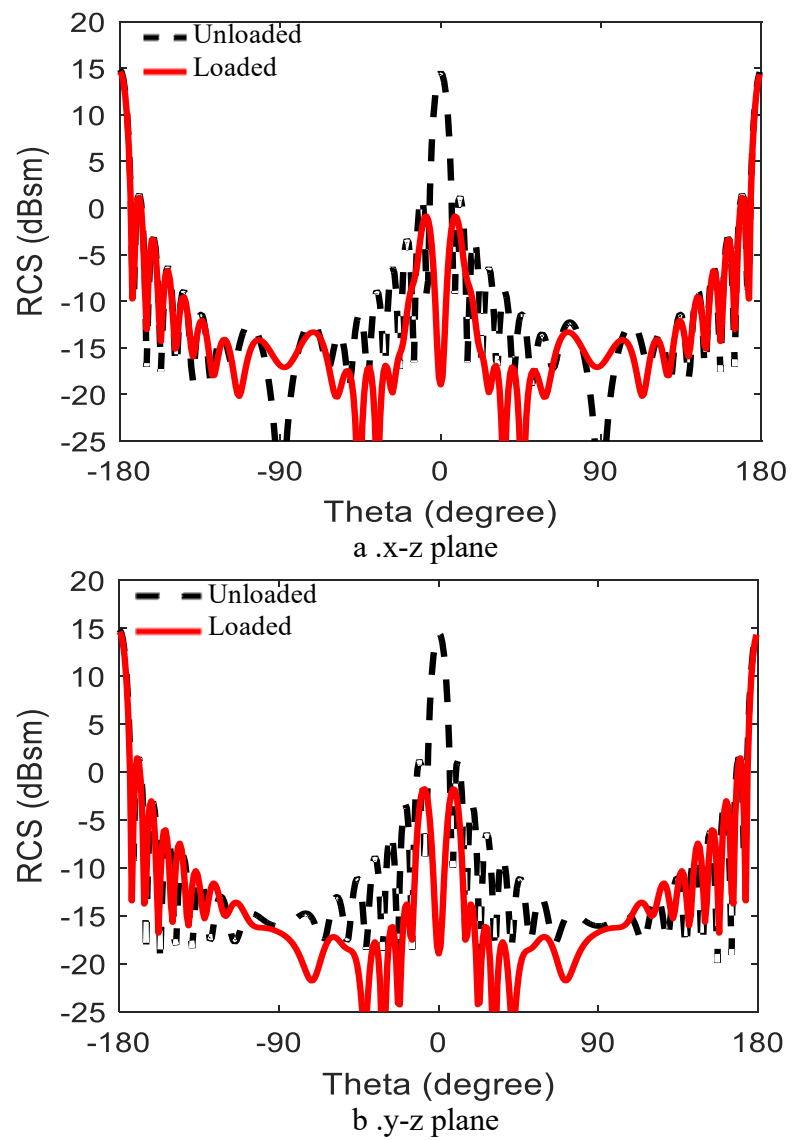

Fig. 10. The RCS variation versus angle for two dimension holes tapering off-diagonal with $\alpha=0.18, R_{0}=2.64 \mathrm{~mm}$, and $11.75 \mathrm{GHz}$.

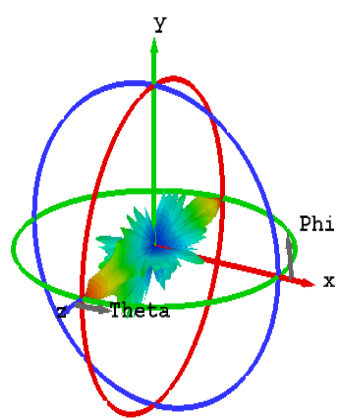

a. $\alpha=0.02$

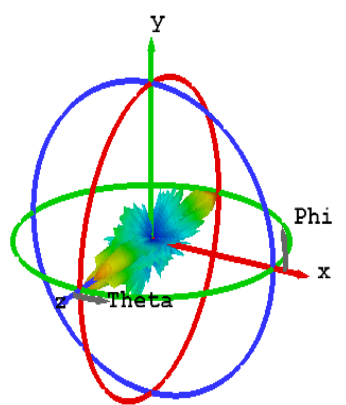

c. $\alpha=0.1$

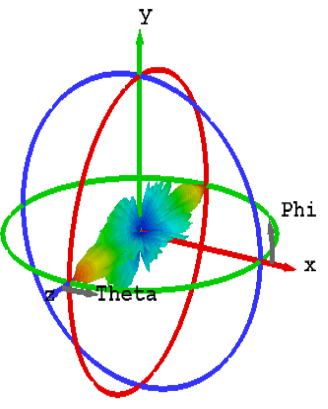

b. $\alpha=0.06$

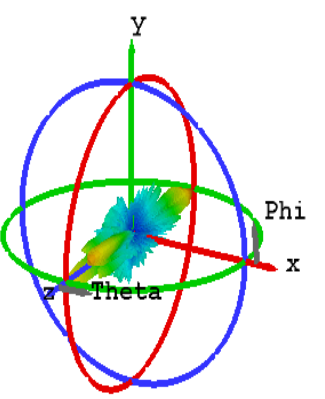

d. $\alpha=0.18$
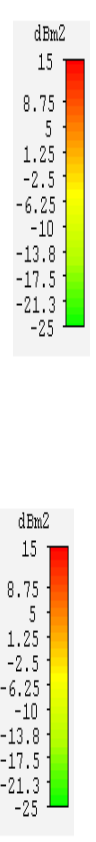

Fig. 11. The 3D RCS variation versus angle for two dimension holes tapering off-diagonal with $R_{0}=2.64 \mathrm{~mm}$, and $11.75 \mathrm{GHz}$. 


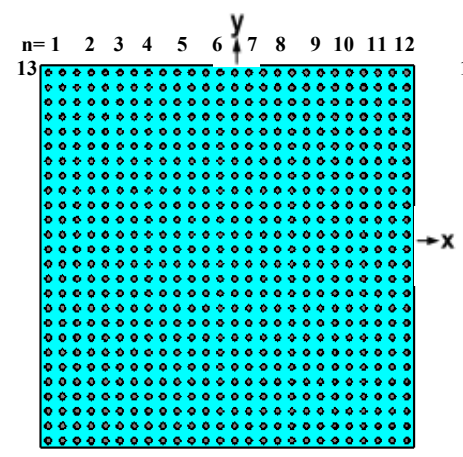

a. $\alpha=-0.02$

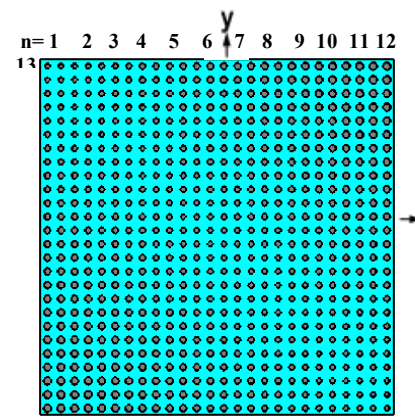

c. $\alpha=-0.1$

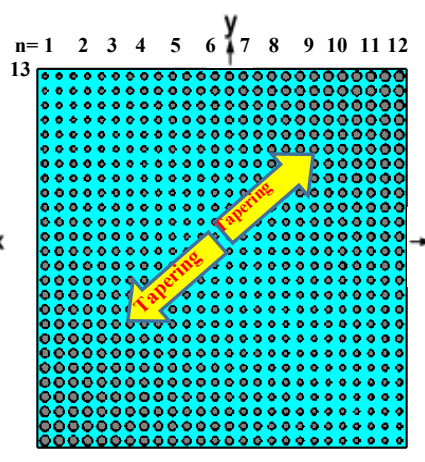

b. $\alpha=-0.06$

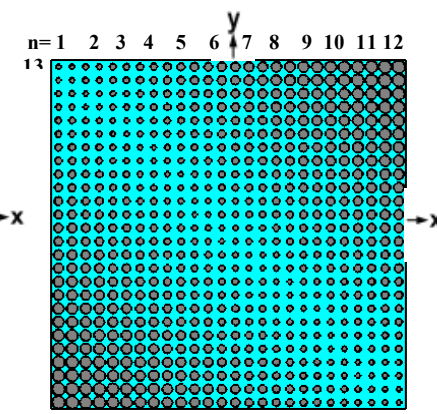

d. $\alpha=-0.18$

Fig.12. The configurations of off-diagonal hole tapering in two directions with different $\alpha, R_{0}=1.5 \mathrm{~mm}, n=1,2 \ldots, 13$ and $\zeta=1$.

When the hole radius of the reference diagonal is small and increases with the subsequent off-diagonals, the second two directions tapering is produced. The configurations of diagonal tapered holes in two directions with $R_{0}=1.5 \mathrm{~mm}, \mathrm{~S}_{\mathrm{p}}=7.5 \mathrm{~mm}, n=1$, $2 \ldots, 13$ and $\zeta=1$ at different values of, $\alpha$, are shown in Fig. 12. The holes' radii are increased away from the main diagonal and are kept constant on the same diagonal. A reduction in RCS is achieved in the band from 11 to $13 \mathrm{GHz}$ for different scaling coefficient values as plotted in Fig. 13. The minimum RCS value by $31 \mathrm{~dB}$ is achieved for $\alpha=-0.18$ at $11.75 \mathrm{GHz}$. The RCS pattern for $\alpha=-0.18$ at $11.75 \mathrm{GHz}$ in different planes is shown in Fig. 14 . A symmetrical pattern is obtained with wide angle RCS reduction from $-111^{\circ}$ to $111^{\circ}$ degrees. 3-D RCS scattering patterns for different scaling factor $\alpha$ at $11.75 \mathrm{GHz}, R_{0}=1.5 \mathrm{~mm}, \mathrm{~S}_{\mathrm{p}}=7.5 \mathrm{~mm}$, and $\zeta=1$ are illustrated in Fig. 15.

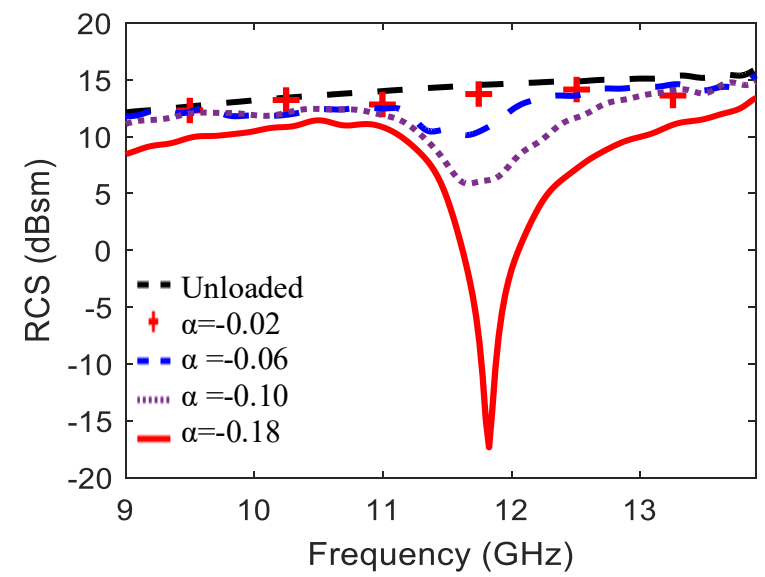

Fig. 13. The RCS versus frequency for two dimension holes tapering off-diagonal with different $\alpha, R_{o}=1.5 \mathrm{~mm}$.
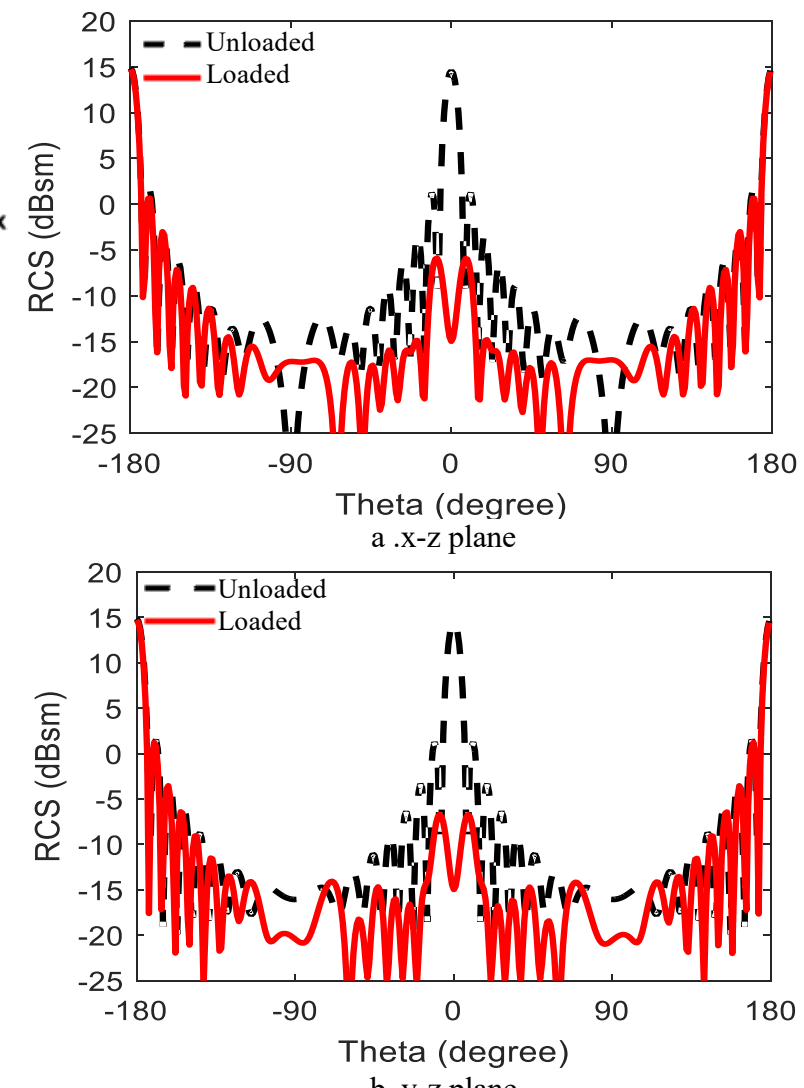

Fig. 14. The RCS variation versus angle for two dimension holes tapering off-diagonal for $\alpha=-0.18, R_{0}=1.5 \mathrm{~mm}$, and $\zeta=1$ at 11.75 $\mathrm{GHz}$ in different planes.
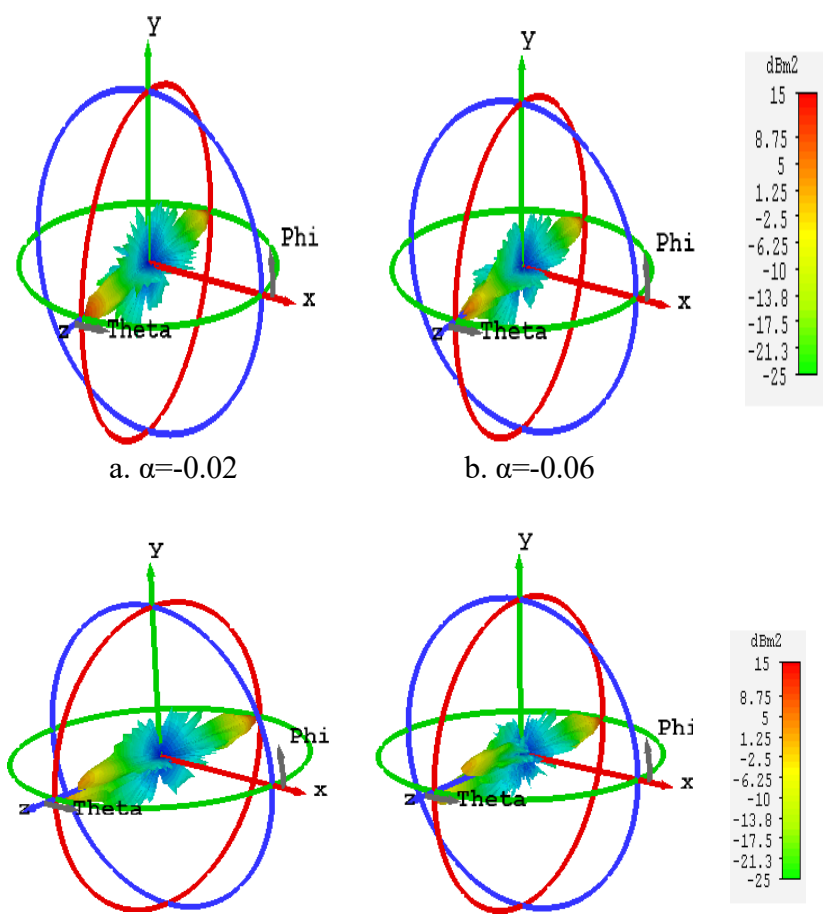

c. $\alpha=-0.1$

d. $\alpha=-0.18$

Fig. 15. The 3-D RCS for two dimension holes tapering offdiagonal for $\alpha=-0.18, R_{0}=1.5 \mathrm{~mm}$, and $\zeta=1$ at $11.75 \mathrm{GHz}$ in different planes. 


\section{Conclusion}

The RCS reduction of metallic sheet is investigated by loading it with a dielectric sheet with different configurations of drilled via holes. The first approach studies a decrease in the RCS by loading the metallic plate with tapered graded perforated dielectric sheet in a single direction. The graded perforations add a progressive phase shift on the incoming waves to be deflected from the normal direction. The RCS reduction occurs in the band from 11.5 to $13.5 \mathrm{GHz}$ for various scaling coefficient values. A maximum RCS value of $13 \mathrm{~dB}$ is done for $\alpha=-0.18$ at 11.75 $\mathrm{GHz}$, with wide angle $\mathrm{RCS}$ reduction from $\pm 130^{\circ}$ degrees. The RCS reduction in the band from 11 to $12.75 \mathrm{GHz}$ is achieved for off-diagonal holes tapering in two directions for various scaling coefficient values. A maximum RCS reduction of $33.5 \mathrm{~dB}$ is obtained with $\alpha=-0.18$ at 11.75 $\mathrm{GHz}$. Different reference hole radii are selected to minimize the RCS.

\section{References}

[1] E.F. Knott, Radar Cross Section, 2nd Edition, Springer Science \& Business Media, 2012.

[2] G.T. Ruck, D.E. Barrick, W.D. Stuart, are C.K. Krichbaum, Radar cross section handbook, vol. 1, pp. 370-371, New York: Plenum press, 1970.

[3] H. Singh, R.M. Jha, Active Radar Cross Section Reduction: Theory and Applications, Cambridge University Press; 1st Ed., 2015.

[4] D. Pozar, "Radiation and scattering from a microstrip patch on a uniaxial substrate," IEEE Transactions on Antennas and Propagation, vol. 35, no. 5, p. 613-621, 1987.

[5] H. Xu, H. Zhang, G. Li, Q. Qu, and K. Lu, “An ultrawideband fractal slot antenna with low backscattering cross section," Microwave and Optical Technology Letters, vol. 53, no. 5, p. 1150-1154, 2011.

[6] F. Costa, S. Genovesi, A. Monorchio, "A frequency selective absorbing ground plane for low-RCS microstrip antenna arrays," Progress in Electromagnetics Research, vol. 126, p. 317-332, 2012.

[7] Edalati, Arezou, and Kamal Sarabandi. "Wideband, wide angle, polarization independent RCS reduction using nonabsorptive miniaturized-element frequency selective surfaces." IEEE Transactions on Antennas and Propagation, vol. 62, no. 2, pp. 747-754, 2014.

[8] A.K. Bhattacharyya, "Radar cross section reduction of a flat plate by RAM coating." Microwave and Optical Technology Letters, vol. 3, no. 9, pp. 324-327, September 1990.

[9] F. Wang, S. Gong, S. Zhang, "Broadband RCS reduction of antenna with AMC," In IEEE 4th AsiaPacific Conference onAntennas and Propagation (APCAP), pp. 606-607, 2015.

[10] H. Singh, S. Antony, R.M. Jha, Plasma-based Radar Cross Section Reduction, Springer Singapore Heidelberg New York, 2016.

[11] S.H. Zainud-Deen, S.M. Gaber, Hend A. Malhat, and K. H. Awadalla, "Perforated Nanoantenna Reflectarray," Progress In Electromagnetics Research M, PIER M, vol. 29, pp. 253-265, January 2013.
[12] S.H. Zainud-Deen, Hend A. Malhat, and K.H. Awadalla,"8x8 Near-field focused circularly polarized cylindrical DRA array for RFID applications," Electrical and Electronic Engineering Journal, 5-11, October 2011.

[13] Hend A. Malhat, and S.H. Zainud-Deen, "Low-profile quad-band perforated rectangular dielectric resonator antenna for wireless communications," IET The Journal of Engineering, vol. 1, no. 8, pp. p. 448 - 451, August 2017.

[14]R. Marklein, "The finite integration technique as a general tool to compute acoustic, electromagnetic, elastodynamic, and coupled wave fields," IEEE Press, New York, USA, pp. 201-244, 2002.

[15] M.L. Waller, and S.A. Rao," Application of adaptive basis functions for a diagonal moment matrix solution of arbitrarily shaped three-dimensional conducting body problems," IEEE Transaction on Antennas and Propagation, vol. 50, no. 10, pp. 1445 - 1452, Oct. 2002. 